\title{
Norois
}

Environnement, aménagement, société

$215 \mid 2010 / 2$

Impacts morphogéniques des tempêtes

\section{Franchissement et migration des cordons de galets par rollover. Impact de la tempête du 10 mars 2008 dans l'évolution récente du Sillon de Talbert (Côtes-d'Armor, Bretagne)}

Crestal overtop and gravel barrier migration by rollover. Impact of 10th March 2008 storm on the actual evolution of Sillon de Talbert (Côtes-d'Armor, Brittany)

Pierre Stéphan, Serge Suanez et Bernard Fichaut

\section{(2) OpenEdition}

Journals

Édition électronique

URL : https://journals.openedition.org/norois/3252

DOI : $10.4000 /$ norois.3252

ISBN : 978-2-7535-1563-5

ISSN : 1760-8546

Éditeur

Presses universitaires de Rennes

Édition imprimée

Date de publication : 30 septembre 2010

Pagination : 59-75

ISBN : 978-2-7535-1164-4

ISSN : 0029-182X

Référence électronique

Pierre Stéphan, Serge Suanez et Bernard Fichaut, « Franchissement et migration des cordons de galets par rollover. Impact de la tempête du 10 mars 2008 dans l'évolution récente du Sillon de Talbert (Côtes-d'Armor, Bretagne) », Norois [En ligne], 215 | 2010/2, mis en ligne le 01 septembre 2012, consulté le 14 janvier 2022. URL : http://journals.openedition.org/norois/3252 ; DOI : https://doi.org/ $10.4000 /$ norois.3252 


\title{
FranchisSEMENT ET MigRATION DES CORDONS
}

DE GALETS PAR ROLLOVER

\section{IMPACT DE LA TEMPÊTE DU IO MARS 2008 DANS L'ÉVOLUTION RÉCENTE du Sillon de Talbert (Côtes-d’Armor, Bretagne)}

\author{
Pierre Stéphan, Serge Suanez, Bernard Fichaut \\ Géomer - LETG UMR 6554 CNRS \\ (Université de Bretagne Occidentale), \\ Institut Universitaire Européen de la Mer, Technopole Brest-Iroise, place Nicolas-Copernic - 29280 Pouzané, France \\ pierre.stephan@univ-brest.fr,serge.suanez@univ-brest.fr,bernard.fichaut@univ-brest.fr
}

\section{RÉSUMÉ}

Cette étude analyse dans un premier temps l'impact de la tempête du 10 mars 2008 sur l'évolution récente du Sillon de Talbert correspondant à une flèche de galets située sur la façade septentrionale de la péninsule armoricaine. Dans un second temps, le rôle des épisodes de franchissement dans le recul récent par rollover de ce cordon est étudié à partir d'un suivi topo-morphologique annuel, réalisé au DGPS entre 2002 et 2008, anquel s'ajoute une analyse des conditions météo-marines enregistrées durant cette période. Le 10 mars 2008, la forte agitation liée à la tempête Johanna coüncide avec une marée de vive-eau de coefficient 106, entraînant un franchissement important du Sillon de Talbert par les vagues. Cet épisode météo-marin extrêmement morphogène se solde par un transfert massif de sédiments en direction du revers et un recul du cordon d'une dizaine de mètres en moyenne. Ce recul particulièrement brutal marque la fin d'une phase de stabilité enregistrée entre 2002 et 2007, caractérisée par l'absence d'épisode de franchissement significatif. La tempête Johanna est suivie d'une période de reconstitution relativement rapide de la crête et une tendance à l'exhaussement du cordon est observée sous des conditions météomarines plus clémentes entre les mois de mars et de septembre 2008. Cette réponse traduit ainsi une bonne résilience du cordon à l'impact d'événements brutaux. Enfin, les résultats mettent en évidence plusieurs phases d'activité morphogène sur le Sillon de Talbert. Ces phases sont également reconnues sur d'autres secteurs littoraux de Bretagne et s'inscrivent dans des cycles décennaux d'évolution des rivages qui pourraient être associés aux variations pluri-annuelles de l'indice hivernal d'oscillation nord-atlantique.

MotS CLÉ : Cordon de galets - franchissement - rollover - flèche littorale - Bretagne - tempête - érosion - suivi topo-morphologique - DGPS - niveau marin - niveau d'eau

\section{ABSTRACT}

Crestal overtop and gravel barrier migration by rollover. Impact of 10th March 2008 storm on the actual evolution of Sillon de Talbert (Côtes-d'Armor, Brittany)

This research concerns the morphological impact caused by the storm Johanna (10 Marsh 2008) on the recent evolution of Sillon de Talbert, a swash-aligned gravel spit located in 
the north of Brittany. The role played by overwash events on gravel barrier rollover processes between 2002 and 2008 is also studied by analysing topographic and oceanographic data set collected in Sillon de Talbert coastal area. In March 2008, the high tide levels associated to stormy wave conditions caused the flooding of the spit which migrated landward $10 \mathrm{~m}$. This erosional phase occurred after a long period of stability related to a low morphogenic activity between 2002 and 2007. After the storm Johanna, a crestal overtoppping phase is measured between March and September 2008 showing the great resilience of the barrier to an extrem event. This results show different phases of morphogenic activity on the Sillon de Talbert, also recognised on other coastal areas along the Brittany coastline, which could be related to hivernal NAO index oscillations.

KEY WORDS : gravel barrier - overwash - rollover - spit - Brittany - storm - erosion topo-morphological survey-DGPS - sea-level-water level

Les travaux portant sur la dynamique générale des cordons de galets ont mis en évidence leur évolution par rollover, mécanisme entraînant le roulement progressif des formes d'accumulation sur elles-mêmes et leur recul vers l'intérieur des terres (Carter et al., 1987, 1989; Carter et Orford, 1993; Forbes et al., 1991, 1995; Héquette et Ruz, 1991; Orford et al., 1991 ; 1995). Le mécanisme de rollover se caractérise par un déplacement de matériel sédimentaire issu de la face externe vers le revers du cordon. Ce transfert sédimentaire s'effectue le plus souvent de manière épisodique et suppose l'intervention d'événements météo-marins au cours desquels les plus hauts jets de rive franchissent le sommet des accumulations. En contexte macro-tidal, ce franchissement s'effectue généralement lorsqu'une pleine mer de vive-eau coïncide avec le passage d'un fort coup de vent ou d'une tempête près des côtes.

L'impact des épisodes de franchissement sur la morphologie des cordons de galets a été décrit en détail par J. D. Orford et R. W. G. Carter (1982) et J. D. Orford et al. (1991), sur la base de simples observations de terrain. Quatre types de réponse morphologique ont été identifiés selon l'importance du franchissement (fig. 1). Tout d'abord, lorsque les plus hauts jets de rive atteignent tout juste la crête, les cordons tendent à s'exhausser (phénomène dit d'overtopping par les anglosaxons). L'infiltration des jets de rive dans les interstices du sédiment diminue la compétence de la nappe de retrait et explique cette tendance à l'accrétion sommitale (Holman et Sallenger, 1985; Butt et Russel, 2000; Masselink et Li, 2001 ; Buscombe et Masselink, 2006). Ensuite, dans un contexte de léger débordement des cordons par les plus hauts jets de rive, la crête est érodée et s'abaisse topographiquement. Généralement dans ce cas, le volume de matériel déversé sur le revers n'est pas suffisant pour entraîner un recul significatif des accumulations par rollover. C'est donc uniquement lors d'événements météo-marins associant de très hauts niveaux d'eau et des vagues de tempête que toute la partie haute des cordons est susceptible de se déverser sur le revers. Dans ce contexte l'écrêtement s'accompagne d'un transfert important de galets en direction du revers permettant un recul significatif des cordons par rollover. J. D. Orford et al. (1991) rappellent que, dans ces conditions, la rupture des cordons est également possible.

Dans la continuité de ces travaux, D. L. Forbes et al. (1995) ont insisté sur les effets de seuil qui caractérisent le mécanisme de rollover. Les auteurs ont montré que le fonctionnement morphodynamique non linéaire des cordons de galets, dont la mobilité se marque par des périodes de stabilité plus ou moins longues, est caractérisé par de courtes phases d'érosion brutale et de recul liées uniquement à l'intervention d'événements météo-marins importants, voire extrêmes. Malgré l'intérêt que représente l'analyse fine de tels épisodes morphogènes pour la gestion de la mobilité des cordons de galets, les recherches menées sur le sujet sont peu nombreuses jusqu'à présent. Cette lacune tient probablement à la difficulté de cerner de tels épisodes dont l'occurrence est généralement très faible. 


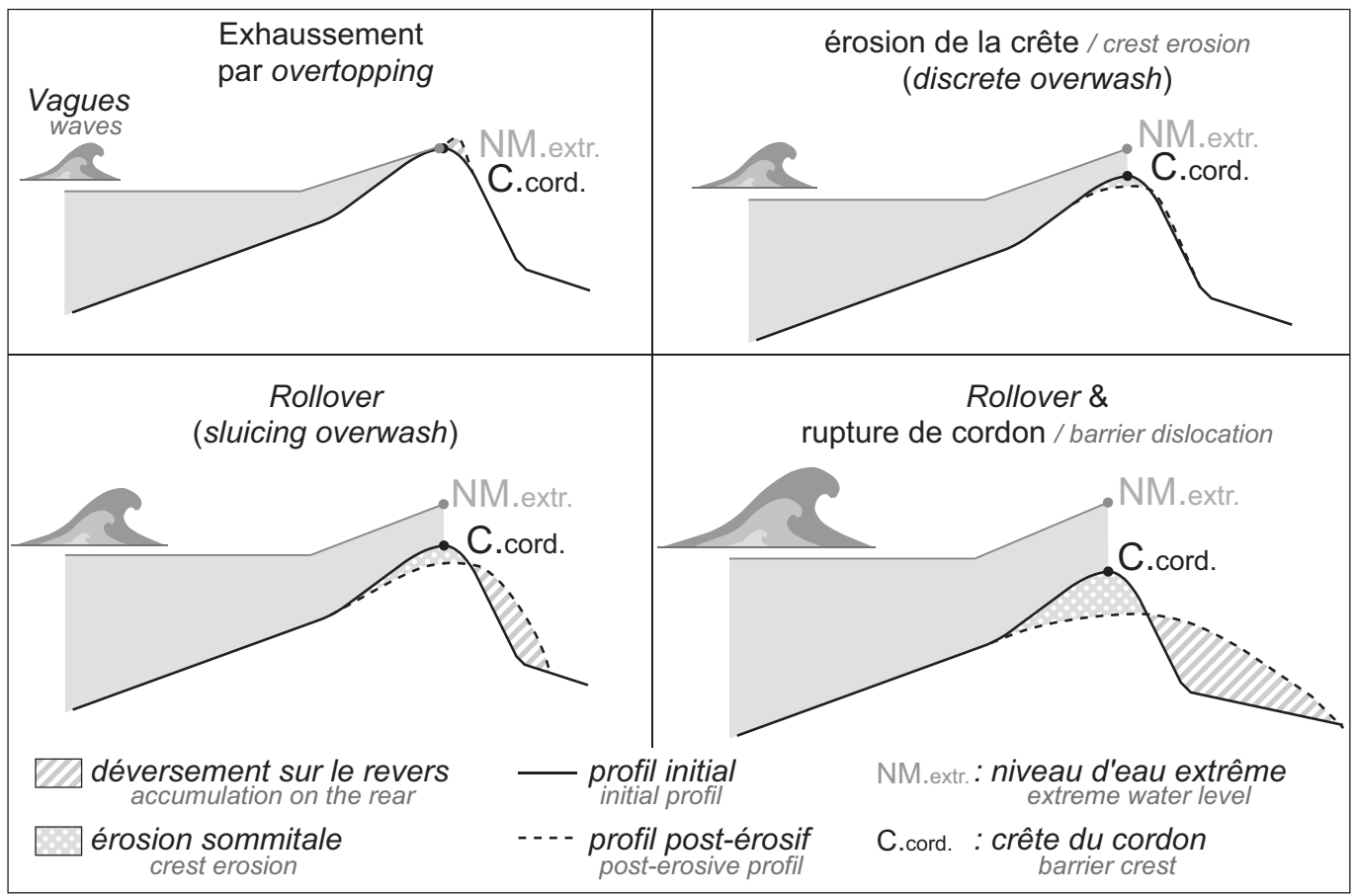

Figure 1 : Réponses morphologiques des cordons de galets selon l'importance du franchissement (d'après J. D. Orford et R. W. G. Carter, 1982).

Morphological responses associated to overwash conditions on a gravel barrier

La tempête Johanna, du 10 mars 2008, constitue l'un des événements les plus morphogènes de ces dernières années le long des côtes de Bretagne (Cariolet et al., ce volume). La conjonction d'une forte agitation marine avec une marée de vive-eau a réuni les conditions météo-marines favorables au franchissement des accumulations littorales par les plus hauts jets de rive et, dans le cas des cordons de galets, à leur recul par rollover.

Cet article se propose donc d'examiner l'impact de cet événement extrême sur la mobilité du Sillon de Talbert, flèche de galets située sur la façade septentrionale de Bretagne, et de replacer cet épisode au sein d'une évolution pluriannuelle par le biais d'un suivi topo-morphologique effectué au DGPS entre 2002 et 2008. Parallèlement, cette recherche s'est attachée à définir le rôle des épisodes de franchissement dans la mobilité du cordon en reconstituant les conditions météo-marines lors du suivi et en retraçant notamment la chronologie des épisodes morphogènes.

\section{Le secteur d'étude}

Le Sillon de Talbert se situe sur la façade septentrionale de la péninsule armoricaine (fig. 2). Il forme une flèche à pointe libre qui s'allonge en direction du nord-est sur une distance d'environ $3,2 \mathrm{~km}$ et une largeur moyenne de $100 \mathrm{~m}$, avant de s'évaser dans sa partie distale en se recourbant sous la forme d'un crochet adoptant une morphologie, dite en tête d'épingle (photo 1A). Dans son prolongement, l'archipel d'Olonne forme un ensemble d'îlots granitiques reliés entre eux par plusieurs accumulations de galets submersibles à pleine mer (photo 1B). 


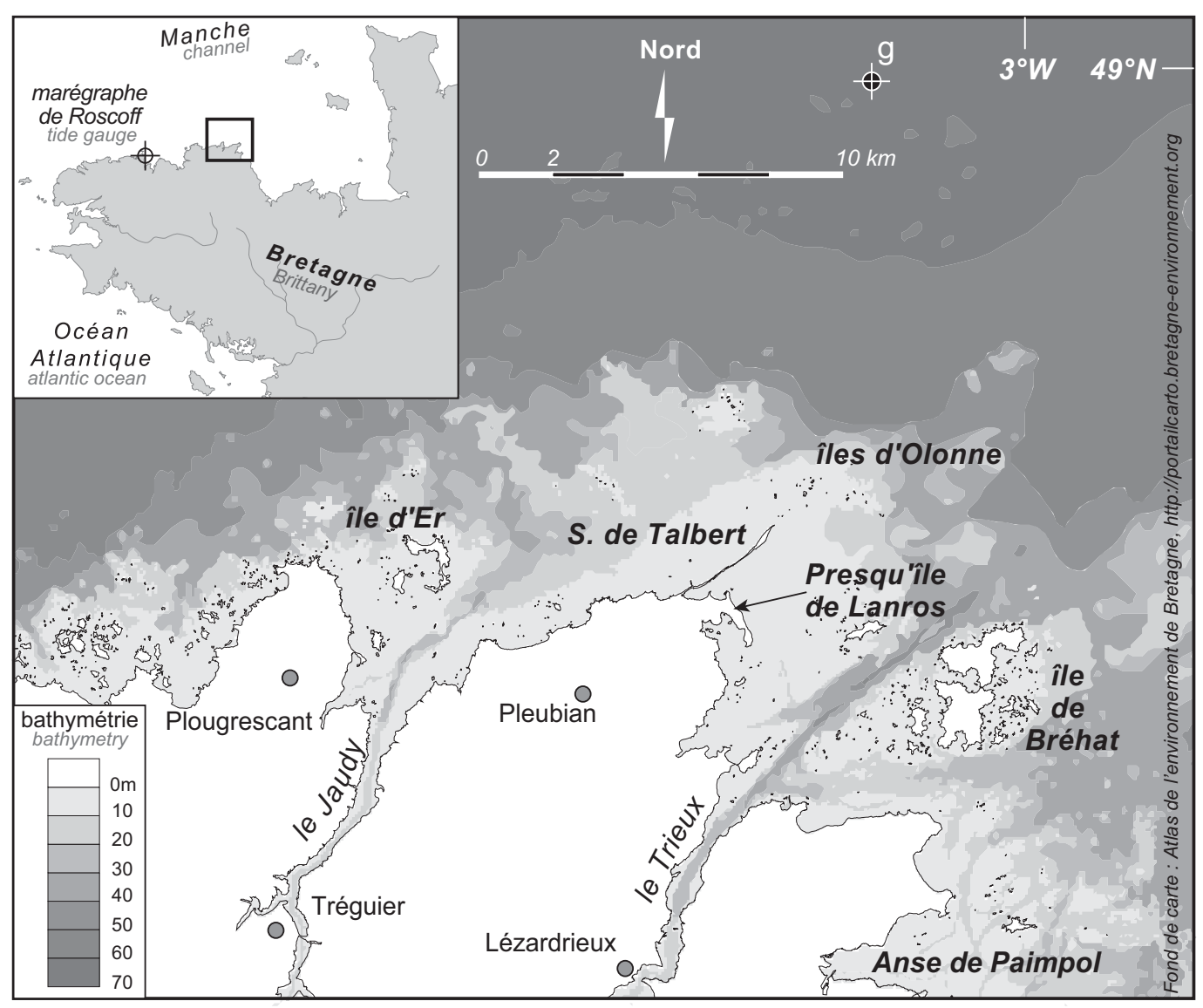

Figure 2 : Localisation du Sillon de Talbert Location maps of Sillon de Talbert

Ce cordon représente actuellement un volume sédimentaire estimé à $1,23.10^{6} \mathrm{~m}^{3}$ qui se compose de sables, graviers et galets en proportion variable le long du cordon. Quatre unités morphosédimentaires peuvent être distinguées à partir de la racine jusqu'à la pointe de la flèche (fig. 3 - planche V). La première unité, essentiellement sableuse, s'étend sur une distance de $500 \mathrm{~m}$ et présente une faible pente (comprise entre $5 \%$ et $12 \%$ ). La crête de l'accumulation est coiffée par une dune dont l'altitude dépasse par endroits 14 m CM (Cote Marine). Cette section bénéficie de la protection de nombreux écueils sur le platier rocheux. La seconde unité morphosédimentaire correspond à la partie proximale du cordon et se compose d'un mélange très hétérométrique de sables et de galets mal triés (médiane des galets : 52 mm, indice de tri : 1,15) (Morel, 1997). Le cordon montre une pente douce (entre $5 \%$ et $7 \%$ ) et un profil très surbaissé dont le sommet atteint à peine l'altitude de 11,5 m CM (fig. 3 - planche V).

La troisième unité correspond à la partie médiane du cordon, essentiellement composée de galets (médiane des galets : 2,6 mm) (Morel, 1997). L'accumulation présente un profil nettement plus redressé et son altitude atteint en moyenne 12,5 m CM (fig. 3 - planche V). Enfin, la quatrième unité morphosédimentaire forme la partie distale du Sillon de Talbert qui se termine par une série de crochons rabattus sur le revers du cordon par la diffraction. Du point de vue morphodynamique, c'est dans ce secteur que l'aspect réfléchissant du cordon est le plus 

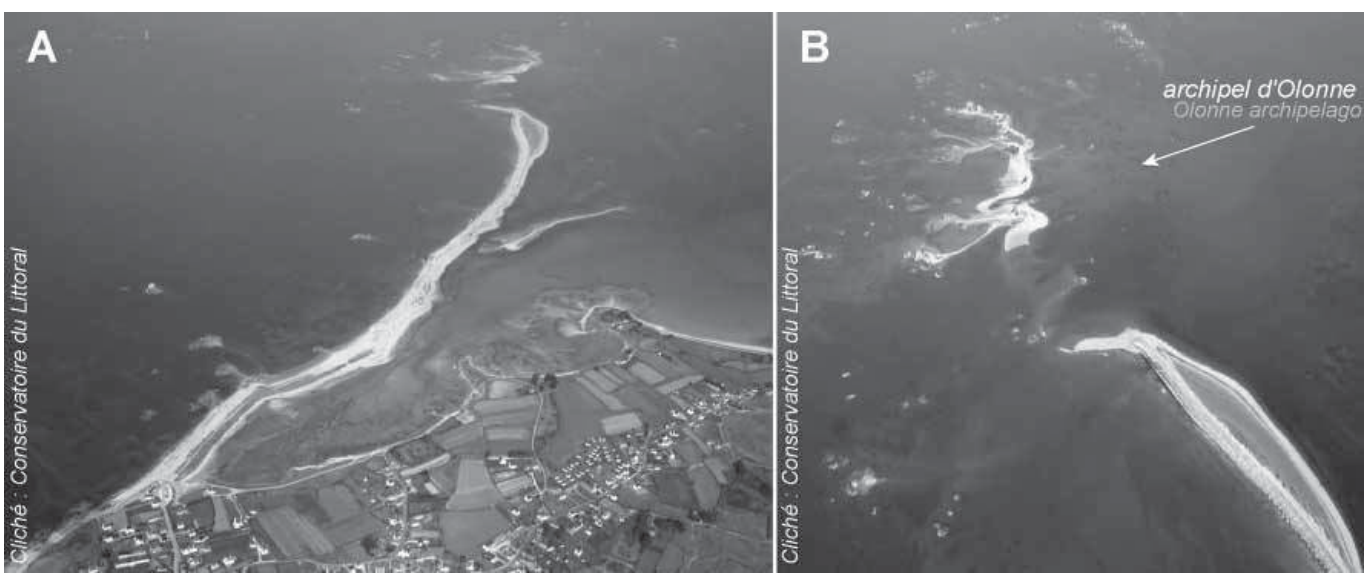

Photo 1 : Vues aériennes du Sillon de Talbert (mars 2007)

A : Sillon de Talbert dans son ensemble. B : partie distale du Sillon et archipel d'Olonne. Aerial views of Sillon de Talbert

accentué. La pente de la plage est de $15 \%$ en moyenne et la crête se situe à une altitude de 13 m CM (fig. 3 - planche V).

L'ensemble du secteur est situé en contexte macrotidal, avec un marnage maximum de 10,85 m. Les houles dominantes proviennent essentiellement de l'ouest-nord-ouest. Les hauteurs significatives $\left(\mathrm{H}_{\text {sig }}\right)$ sont comprises entre 1 et $1,5 \mathrm{~m}$, les périodes $\left(\mathrm{T}_{\text {pic }}\right)$ entre 9 et 10 secondes. Lors des épisodes tempétueux, la hauteur maximum des vagues peut toutefois dépasser $9 \mathrm{~m}$ et la période 20 secondes (Stéphan, 2008a). Dans ces conditions, le Sillon de Talbert joue un rôle de barrière naturelle. Il offre ainsi une situation d'abri aux îles de l'archipel de Bréhat à l'est (fig. 2) et évite la submersion par les vagues de la Presqu'île de Lanros au sud (fig. 2) dont une grande partie (environ $10 \mathrm{ha}$ ) est située sous le niveau des plus hautes mers astronomiques. Par ailleurs, son orientation générale par rapport aux directions dominantes des houles implique un faible transport longitudinal vers sa partie distale, l'essentiel du transport sédimentaire s'effectuant de manière transversale, de la plage en direction du revers.

L'analyse diachronique de cartes anciennes et de photographies aériennes a montré que le Sillon de Talbert connaît un recul par rollover estimé à environ 0,5 m/an depuis 1770 (Pinot, 1994). Cette dynamique régressive, particulièrement rapide au cours des trois derniers siècles, trouve son origine dans une situation de pénurie sédimentaire, résultant de la faiblesse des apports récents en matériel grossier. En effet, pour son alimentation, le cordon a essentiellement bénéficié de l'érosion d'un cordon fossile d'âge éémien dont il ne reste aujourd'hui que quelques plaquages sur l'estran.

Afin de freiner ce recul et d'éviter l'ouverture de brèches, plusieurs interventions de stabilisation ont été entreprises sur le cordon au cours des cinquante dernières années. La plus remarquable d'entre elles a été l'édification en 1974, d'une digue frontale en enrochements longue d'environ $2 \mathrm{~km}$. Toutefois, les fortes tempêtes hivernales qui ont marqué la décennie 1980 ont provoqué un affaissement considérable du cordon. De nouvelles brèches se sont ouvertes dans les années 1990 tandis que le recul du cordon s'est poursuivi en dépit des enrochements (Morel, 1997; Pinot, 1994). En 2001, la gestion du Sillon de Talbert a été confiée au Conservatoire du Littoral, optant pour la suppression de la digue frontale en enrochements dont les blocs ont été concassés (photo 2A), avant d'être disposés en arrière de la flèche sous la forme de trois cordons artificiels servant d'ados (photo 2B). Le matériel concassé a aussi servi à colmater artificiellement deux larges brèches ouvertes dans la partie proximale de la flèche. Parallèlement, une surveillance régulière de l'évolution littorale a été mise en place sous la forme d'un suivi topo-morphologique dont nous présentons ici les résultats. 
Depuis janvier 2007, le Sillon est classé Réserve Naturelle Régionale en raison entre autres de la « particularité » géomorphologique qu’il représente à l'échelle de la Bretagne.

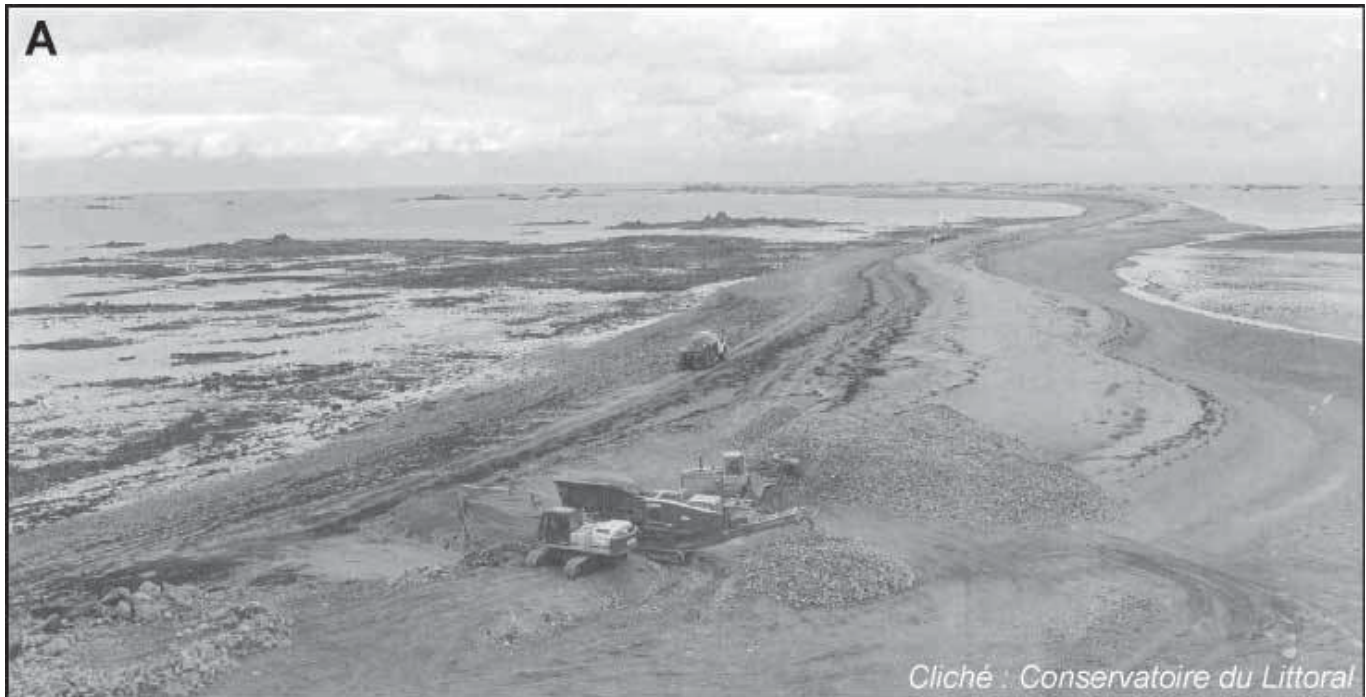

B

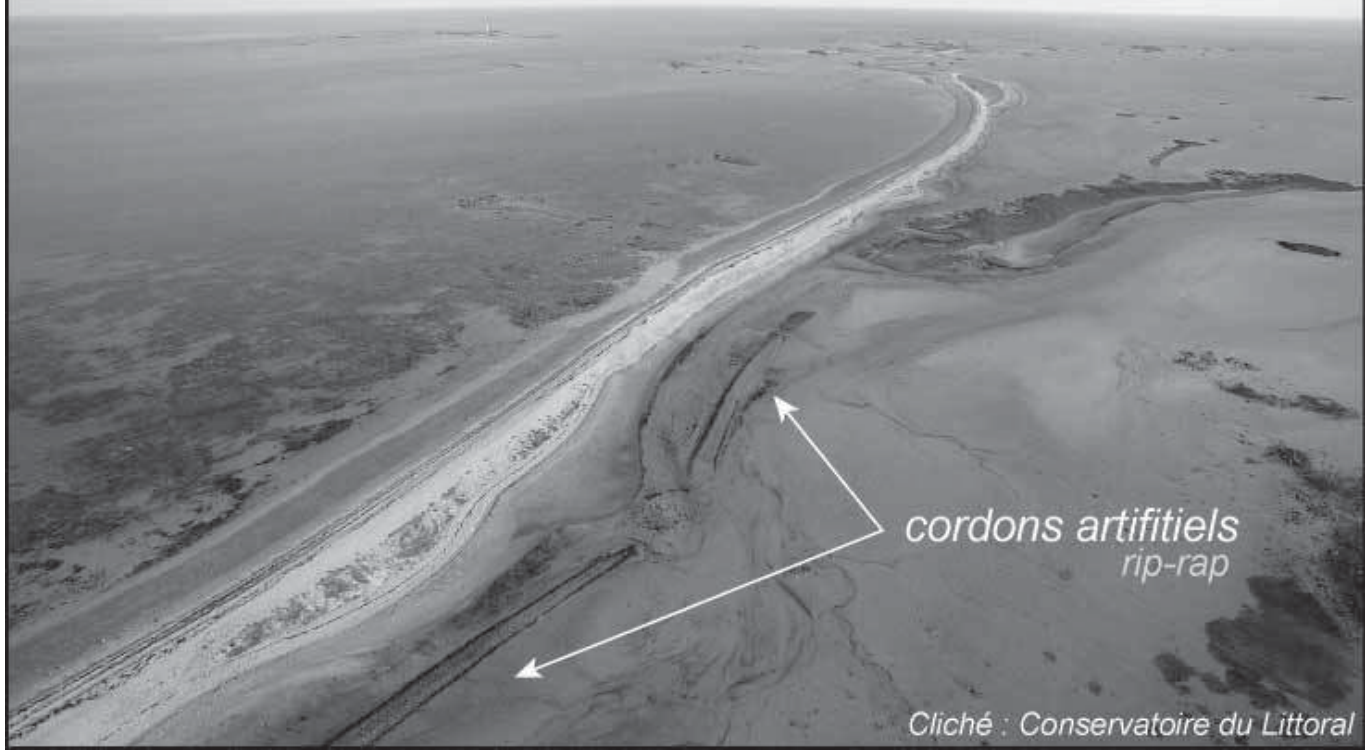

Photo 2 : Vues aériennes du Sillon de Talbert durant les travaux de suppression de la digue frontale en enrochement (A) et après la mise en place des cordons artificiels en arrière de la flèche (B)

Aerial views of Sillon de Talbert during the suppression of the rip-rap along the spit (A) and after the construction of the artificial accumulations behind the spit (B) 


\section{Méthodologie}

Le rôle du franchissement par les vagues de tempête dans la mobilité du Sillon de Talbert a été étudié à partir d'un suivi topo-morphologique couplé à l'analyse des conditions météo-marines et plus particulièrement, celle des plus hauts niveaux d'eau à la côte.

\section{LE SUIVI TOPO-MORPHOLOGIQUE DU SILLON}

Le suivi topo-morphologique repose sur sept campagnes de mesure topographique réalisées entre 2002 et 2008. A l'exception des données obtenues au mois d'octobre 2002 à partir d'un levé Lidar dont la précision altitudinale a été estimée à $10 \mathrm{~cm}$ (Boersma et Hoenderkamp, 2003), toutes les mesures topographiques ont été réalisées à l'aide d'un DGPS en mode cinématique (RTK), avec une précision horizontale et verticale respective de $2 \mathrm{~cm}(\mathrm{x}, \mathrm{y})$ et de $1,5 \mathrm{~cm}(\mathrm{z})$ (Suanez et al., 2008). Les mesures ont été raccordées au système géodésique français à partir d'une borne IGN située à proximité du Sillon de Talbert. Le système de géoréférencement qui a été retenu correspond au Lambert II étendu et les altitudes ont été raccordées dans un premier temps au système IGN69, avant d'être converties en cotes marines (soit +5,12 m par rapport à la cote NGF). Le traitement des données a été réalisé sous le logiciel Surfer 8.0. La méthode du krigeage avec intégration de lignes de rupture a été retenue comme modèle d'interpolation pour la construction de Modèles Numérique de Terrain (MNT) de résolution métrique. La comparaison des différents MNT a permis d'obtenir à la fois les valeurs de recul ou d'avancée du cordon, mesurées à la base du revers, et les variations altitudinales de la crête, en termes d'exhaussement et/ou d'abaissement. Ces mesures ont été réalisées le long de 110 transects disposés perpendiculairement au cordon et espacés d'une trentaine de mètres les uns des autres.

\section{L'ANALYSE DES CONDITIONS MÉTÉO-MARINES}

L'analyse des épisodes de franchissement repose sur la reconstitution des hauts niveaux d'eau à la côte entre 2002 et 2008 le long de quatre transects et le croisement de ces données avec la topographie du Sillon de Talbert. Les quatre transects choisis sont jugés représentatifs des quatre unités morphosédimentaires qui composent la flèche : racine (P006), parties proximale (P048), médiane (P075) et distale (P100). Le franchissement peut être défini par la formulation suivante (Stéphan, 2008) :

$$
\Delta_{\text {Subm }}=\text { N.M. }_{\text {extr. }}\left[\mathrm{M}_{\text {pred }}+\left(\mathrm{s}^{+} \text {ou s} \mathrm{s}^{-}+\mathrm{R} 2\left(\boldsymbol{\eta}+\mathrm{R}_{\text {swash }}\right)\right]>\mathrm{C}_{\text {cord }}\right.
$$

où $\Delta_{\text {Subm }}$ est la hauteur de la colonne d'eau lors du franchissement, $\mathrm{C}_{\text {cord }}$ est l'altitude de la crête et N.M. ${ }_{\text {extr. }}$ correspond aux niveaux marins extrêmes définis comme la somme des trois composantes intervenant dans la génération de hauts niveaux d'eau à la côte (fig. 4), à savoir :

- la marée prédite $\left(\mathrm{M}_{\text {pred }}\right)$ définie comme l'oscillation quotidienne du plan d'eau liée à la marée astronomique;

- la surcote $\left(\mathrm{s}^{+}\right)$et la décote $\left(\mathrm{s}^{-}\right)$qui s'expliquent à la fois par des variations de pression barométrique et par l'intervention de vents violents poussant les masses océaniques à la côte;

- la somme $\left(\mathrm{R}_{2}\right)$ des phénomènes de setup $(\boldsymbol{\eta})$ et de swash runup $\left(\mathrm{R}_{\text {swash }}\right)$ étroitement dépendants des conditions d'agitation marine au niveau du rivage. Le setup correspond à une déformation du plan d'eau entre le point de déferlement des vagues et la ligne de rivage tandis que le runup est la hauteur du jet de rive atteinte dans la zone de swash.

Les données de marée prédite $\left(\mathrm{M}_{\text {pred }}\right)$ ont été obtenues en hauteurs d'eau horaires par simulation numérique à partir du modèle de marée du SHOM, intégrant une cinquantaine de composantes harmoniques de la marée. Les valeurs de surcote $\left(\mathrm{s}^{+}\right)$et de décote $\left(\mathrm{s}^{-}\right)$ont été calculées à partir des enregistrements marégraphiques du port de Roscoff, distant d'une cinquantaine de kilomètres du 


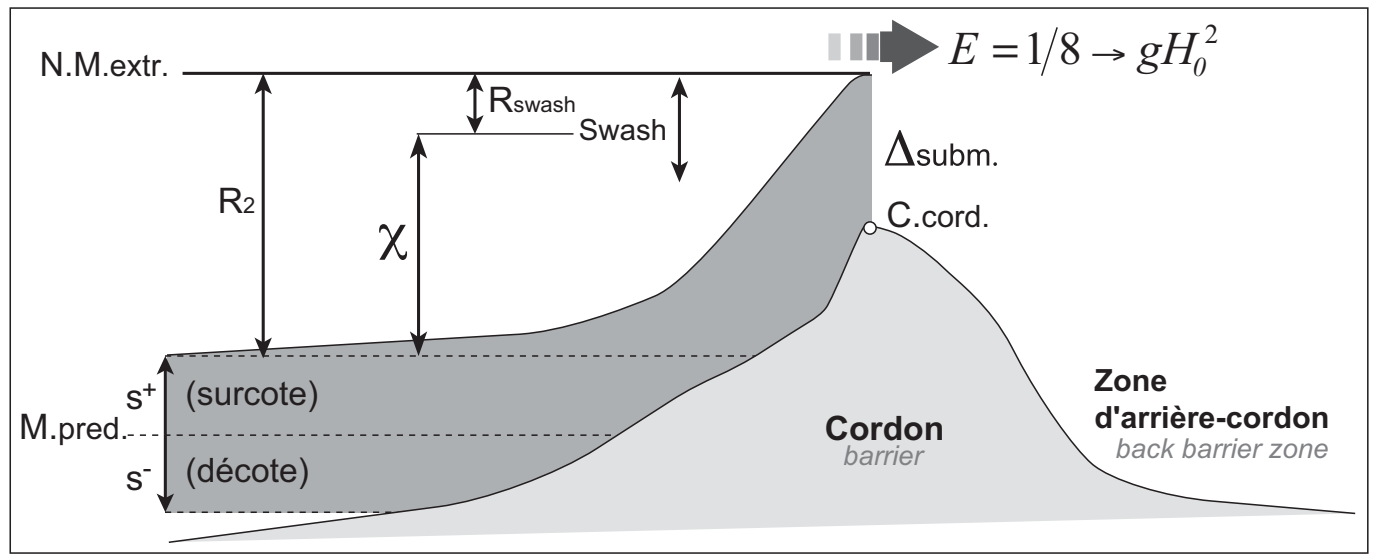

Figure 4 : Paramètres utilisés pour déterminer les hauts niveaux d'eau à la côte et pour hiérarchiser les épisodes de franchissement

Parameters used to define high water levels and to quantify morphological impact of flooding events

secteur d'étude (fig. 2). Cet éloignement induit probablement un certain déphasage et des écarts d'amplitude dans les pics de surcote et de décote dont nous ne pouvons pas tenir compte. Il faut donc considérer ici les données marégraphiques comme une estimation comportant une part d'incertitude non négligeable. Les valeurs de setup $(\boldsymbol{\eta})$ et de sawsh runup $\left(\mathrm{R}_{\text {swash }}\right)$ ont été calculées à partir des équations proposées par H.F. Stockdon et al. (2006) :

$R_{2}=0,043\left(H_{0} L_{0}\right)^{1 / 2}$

$R_{2}=1,1\left(0,35 \beta_{f}\left(H_{0} L_{0}\right)^{1 / 2}+\frac{\left[H_{0} L_{0}\left(0,563 \beta_{f}^{2}+0,004\right)\right]^{1 / 2}}{2}\right)$

$R_{2}=0,73 \beta_{f}\left(H_{0} L_{0}\right)^{1 / 2}$

où, $\mathrm{R}_{2}$ correspond à la hauteur atteinte par $2 \%$ des plus hauts jets de rives, $\boldsymbol{\beta}_{f}$ est la pente calculée sur toute la longueur de la plage, $\mathrm{H}_{0}$ et $\mathrm{L}_{0}$ correspondent respectivement à la hauteur significative et à la longueur d'onde des houles au large. Ces formules empiriques ont été élaborées à partir de mesures de terrain réalisées entre 1982 et 1997 sur dix plages situées en Hollande (Ruessink et al., 1998) et dans les États de Caroline du Nord (Holman, 1986; Holland et Holman, 1993, 1996), de Californie (Holland et al., 1995) et d'Oregon (Ruggiero et al., 2001) aux ÉtatsUnis. La morphologie de ces dix plages et les conditions hydrodynamiques régnant lors de ces campagnes de mesure ont permis de déterminer les phénomènes de setup et de runup pour une large gamme de conditions environnementales. Trois équations sont ainsi proposées en fonction de l'état morphodynamique de la plage, défini par le nombre d'Iribarren $\xi_{0}$ (Battjes, 1974) d'équation:

$$
\xi_{0}=\beta_{f} /\left(H_{0} / L_{0}\right)^{1 / 2}
$$


Pour un état dissipatif $\left(\xi_{0}<0,3\right)$, la formule [2] est utilisée; pour un état intermédiaire $\left(0,3<\xi_{0}<1,25\right)$ il est préconisé d'employer la formule [3] ; la formule [4] s'emploie pour un état réfléchissant $\left(\xi_{0}>1,25\right)$ (Stockdon et al., 2006).

Les paramètres de la houle, nécessaires au calcul du setup et du runup, ont été obtenus par simulation numérique à partir d'un modèle de vagues développé par le service «Vague et Littoral » du SHOM, utilisant les données de vents fournies par le Centre Européen de Prévision météorologique (ECMWF). Sous le logiciel Crest, les vagues ont ensuite été propagées jusqu'au point $\gamma$ de coordonnées $3,046^{\circ} \mathrm{W}$ et $48,964^{\circ} \mathrm{N}$, situé au large de notre secteur d'étude (fig. 2), en emboîtant un modèle de tracé de rayons à plus grande échelle spatiale, tenant compte de la bathymétrie locale.

Les épisodes de franchissement reconnus ont également été hiérarchisés en calculant la densité d'énergie des vagues à la côte lors du franchissement, suivant la formule :

$$
E=1 / 8 \rho g H_{0}^{2}
$$

où E est la somme de l'énergie par unité de surface $\left(\mathrm{J} \cdot \mathrm{m}^{-2}\right)$, $\rho$ est la masse volumique de l'eau (1027 kg/m³ pour une eau salée).

\section{Résultats}

\section{LA TEMPÊTE JOHANNA ET SON IMPACT MORPHOSÉDIMENTAIRE SUR LE SILLON DE TALBERT}

Entre les mois de septembre 2007 et mars 2008, le Sillon de Talbert a connu une érosion considérable (fig. 5). Les transferts sédimentaires, estimés à $120000 \mathrm{~m}^{3}$ (soit $10 \%$ du volume total de l'édifice) se sont opérés essentiellement de la plage vers l'arrière du cordon, témoignant d'une véritable dynamique de rollover. À l'exception de sa racine et de sa partie proximale, la flèche a reculé d'une dizaine de mètres en moyenne vers l'est. Par endroits, le recul du cordon a dépassé $20 \mathrm{~m}$ et s'est accompagné d'un abaissement de la crête de 1,5 m en moyenne (fig. 5).

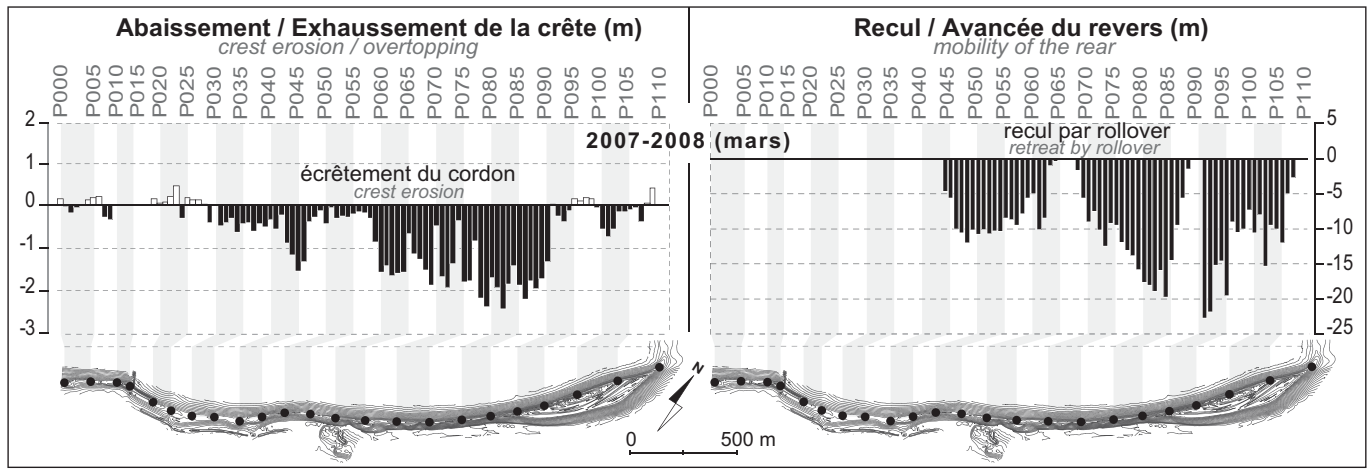

Figure 5 : Évolutions morphologiques mesurées sur le Sillon de Talbert entre les mois de septembre 2007 et de mars 2008

Morphological evolutions of Sillon de Talbert between September 2007 and March 2008

Ces changements morphologiques particulièrement brutaux sont en grande partie le résultat de la tempête Johanna, des 10 et 11 mars 2008. L'analyse détaillée des conditions météo-marines (fig. 6) montre qu'au moins deux épisodes de franchissement majeurs se sont succédés lors du passage de cet événement tempétueux près du Sillon de Talbert. Un premier franchissement est survenu dans la matinée du 10 mars, entre 9 heures et 10 heures (UT+ 1 heure). Des conditions marégraphiques exceptionnelles en sont à l'origine. En effet, la pleine mer de vive-eau de coeffi- 
cient 106 a coïncidé avec un pic de surcote dont les valeurs ont atteint $60 \mathrm{~cm}$ au port de Roscoff (fig. 6). En revanche, les vagues de secteur ouest (direction $280^{\circ} \mathrm{N}$ ) n'ont pas été particulièrement fortes, même si leur hauteur au large a pu atteindre 5,2 m. Ce contexte a toutefois suffi à entraîner une première submersion du cordon par les plus hauts jets de rive et un écrêtement important de l'édifice. Par la suite, un deuxième épisode de franchissement est survenu dans la soirée du 10 mars et a duré entre trois et quatre heures. Cette fois, les vagues d'ouest (direction $290^{\circ} \mathrm{N}$ ) ont été nettement plus fortes que dans la matinée et leur hauteur a atteint 9,5 m au large du Sillon (fig. 6). C'est donc probablement dans ce contexte de très forte agitation marine que s'est opéré l'essentiel des transferts sédimentaires en direction du revers. À l'issue de ces deux épisodes particulièrement morphogènes, les portions les plus fragilisées du Sillon ont pu subir à nouveaux plusieurs épisodes de franchissement durant les phases de pleines mers. En effet, jusqu'au 13 mars, la hauteur des vagues est restée importante (entre 4 et $6 \mathrm{~m}$ ) et les coefficients de marée ont diminué lentement (fig. 6), si bien que les plus hauts jets de rive ont pu franchir à nouveau un cordon déjà largement écrêté.

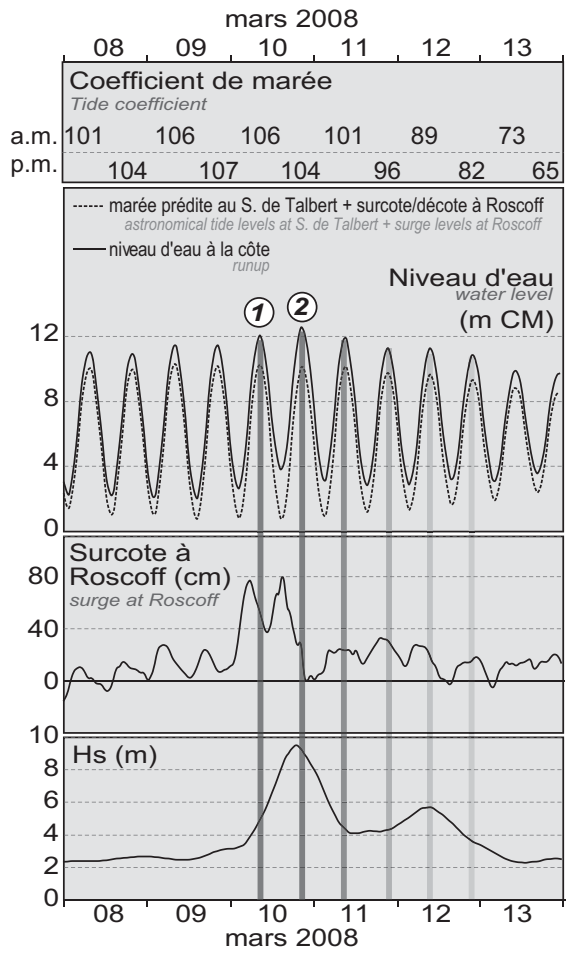

Figure 6 : Conditions météo-marines sur le secteur du Sillon de Talbert entre le 08 et le 13 mars 2008

Tide and wave conditions between 08 and 13 March 2008 on Sillon de Talbert

\section{UN CONTEXTE ANTÉ-TEMPÉTUEUX DE STABILITÉ MORPHOGÉNIQUE}

Le suivi topographique du Sillon de Talbert réalisé depuis 2002 montre que la tempête Johanna a marqué la fin d'une période de relative stabilité morphologique. Entre les mois d'octobre 2002 et de mai 2006, le recul du cordon est resté très ponctuel, de faible ampleur et s'est limité à la partie médiane du Sillon (fig. 7). D’une manière générale, la crête s'est exhaussée sur toute la longueur de la flèche traduisant une consolidation de la partie haute du cordon par le biais des processus dit d'overtopping. L'analyse des conditions météo-marines montre en effet que cette période correspond à une phase de calme morphogénique marquée par l'absence d'épisode de franchissement significatif le long du cordon (fig. 8). Les variations altitudinales de la crête les plus significatives, mesurées entre 2003 et 2005 dans la partie proximale, doivent être attribuées uniquement à la suppression de la digue frontale en enrochement en novembre 2004 et au colmatage de deux brèches ouvertes dans les années 1990.

Certes, entre les mois de mai 2006 et septembre 2007, des modifications morphologiques plus importantes ont été observées (fig. 7). La portion comprise entre les transects P045 et P060 a été largement écrêtée, connaissant également un recul par rollover de 5 à $10 \mathrm{~m}$. À cela s'est ajouté l'ouverture de cinq brèches dans la partie haute du cordon, entre les transects $\mathrm{P} 070$ et P085, provoquant un abaissement de la crête de $1 \mathrm{~m}$ en moyenne. Les données météo-marines indiquent que les principaux changements ont pris place lors des épisodes tempétueux qui ont marqué l'hiver 2006/2007, traduisant ainsi un renforcement des conditions météo-marines tempétueuses durant cette période (fig. 8). Par ailleurs, un recul important du revers a été observé dans la partie distale de la flèche, entre les transects P090 et 


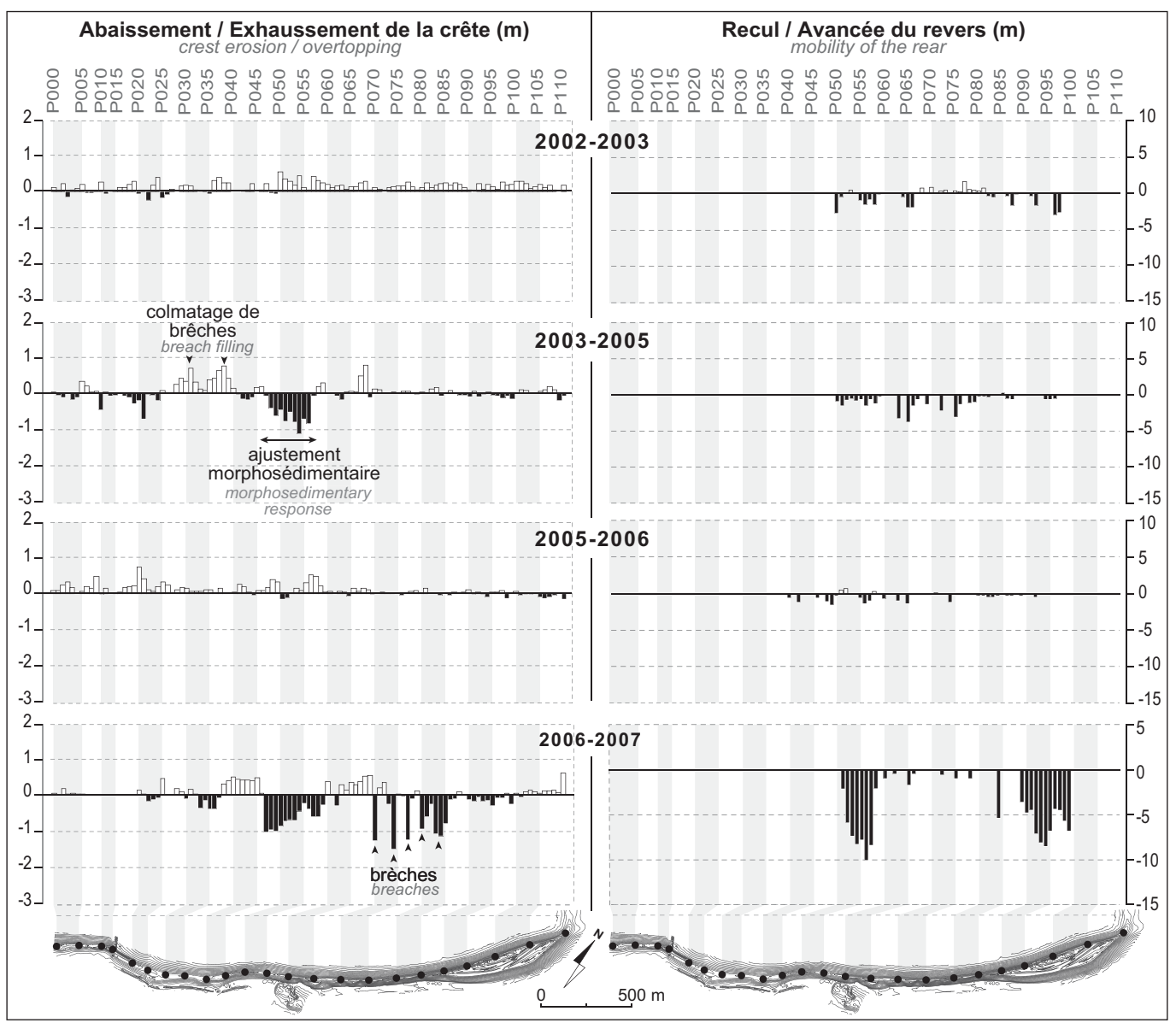

Figure 7 : Évolutions morphologiques mesurées sur le Sillon de Talbert entre les mois d'octobre 2002 et de septembre 2007

Morphological evolutions of Sillon de Talbert between October 2002 and September 2007

P100, et a résulté vraisemblablement de projections de galets liées au déferlement de vagues puissantes sur la plage.

\section{Réponse post-Érosive et résilience du Sillon de Talbert}

Les levés topographiques réalisés au mois de septembre 2008 après la tempête Johanna ont montré que le cordon s'est reconstitué rapidement (fig. 9), sous des conditions météo-marines plus clémentes (fig. 8). La crête du cordon a connu un exhaussement rapide d'environ $50 \mathrm{~cm}$ dans les parties proximale, médiane et distale de la flèche. Ainsi, la période estivale ayant succédé à l'épisode des 10 et 11 mars 2008 semble avoir favorisé un processus de reconstitution progressive de la crête par overtopping. Toutefois, à cela s'est ajouté un recul général du cordon d'environ $2 \mathrm{~m}$ qui s'explique par des épisodes de franchissement désormais plus fréquents, mais peu morphogènes, comme on peut l'observer au niveau des profils P075 et P100 (fig. 8). 


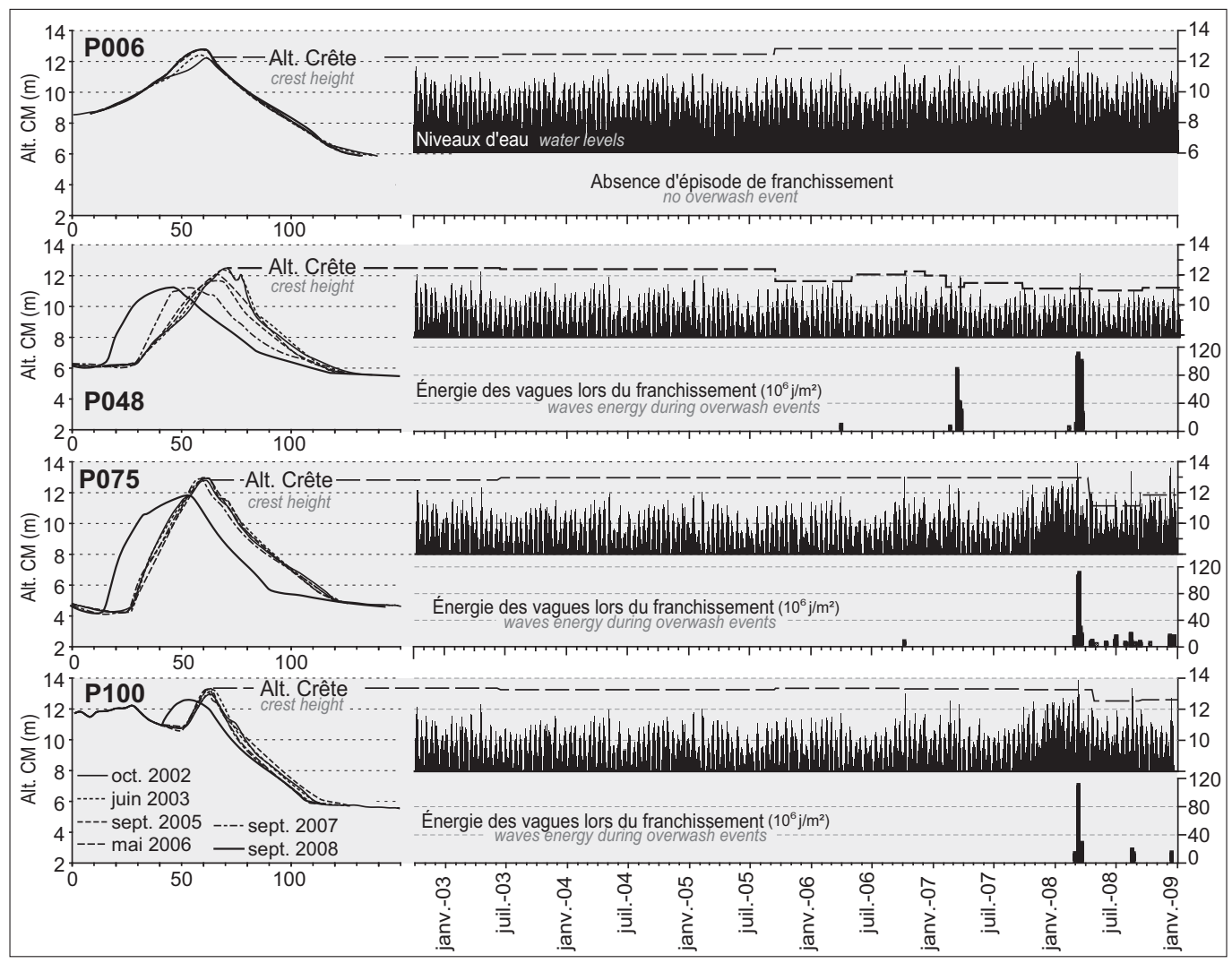

Figure 8 : Chronologie des hauts niveaux d'eau, énergie des vagues lors des épisodes de franchissement et évolutions morphologiques le long des transects P006, P048, P075 et P100

Chronology of high water levels, waves energy during overwash events and morphological evolutions along transects P006, P048, P075 and P100

\section{Discussion}

Lors de l'épisode du 10 mars 2008, deux types de fonctionnement morphodynamique ont pu être observés le long du Sillon de Talbert. Les parties médiane et distale du Sillon de Talbert ont connu une véritable dynamique de roulement de la forme sur elle-même vers l'intérieur des terres (rollover), tandis qu'à la racine du Sillon et dans sa partie proximale, le franchissement s'est simplement traduit par le creusement de couloirs d'érosion à travers la crête, au débouché desquels se sont mis en place des lobes de débordement. Ces réponses différentes s'expliquent en grande partie par la granulométrie du matériel sédimentaire qui constitue les quatre unités morphosédimentaires du Sillon. En effet, les parties médiane et distale sont constituées majoritairement de graviers et de galets, tandis que la fraction sableuse est quasi-inexistante, du moins en surface. Dans ces secteurs, la réponse du cordon au franchissement a donc été identique à celle décrite pour les accumulations de galets stricto sensu (Orford et Carter, 1982). À l'inverse, à la racine et dans la partie proximale du Sillon où domine la fraction sableuse, les changements morphologiques observés s'apparentent à celles décrites sur les cordons sableux de type île-barrière (Leatherman, 1979, 1983; Stockdon et al., 2007). 


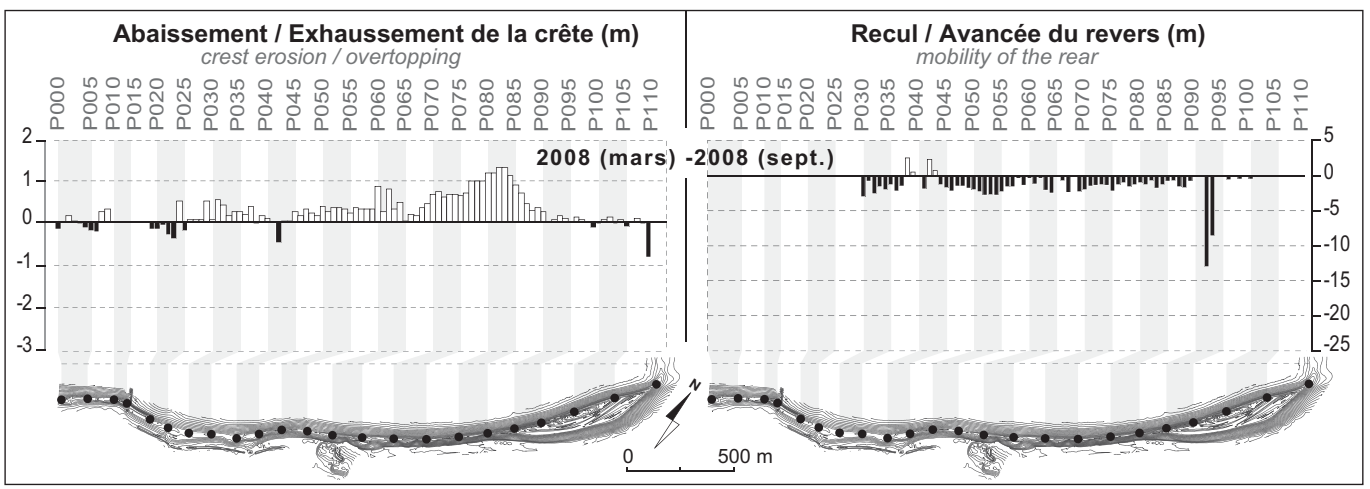

Figure 9 : Évolutions morphologiques mesurées sur le Sillon de Talbert entre les mois de mars 2008 et de septembre 2008

Morphological evolutions of Sillon de Talbert between March 2008 and September 2008

Le renforcement des conditions météo-marines durant l'hiver 2006-2007, suivi par la tempête Johanna du 10 mars 2008, contrastent avec la période de calme hydrodynamique observée entre 2002 et 2006. Ce changement progressif vers des conditions plus morphogènes relève, semble-t-il, de cycles reconnus plus largement à une échelle régionale. Ainsi, la phase de stabilité du Sillon de Talbert observée entre 2002 et 2006 s'inscrit dans une période de faible activité morphogène observée sur de nombreux secteurs littoraux de Bretagne. En rade de Brest, par exemple, les levés topo-morphologiques effectués par P. Stéphan (2008a) sur plusieurs flèches de galets montrent des évolutions mineures de 2003 à 2006. En baie de Saint-Michel-en-Grève, le suivi réalisé par S. Suanez et P. Stéphan (2006) durant cette même période a montré une tendance à l'engraissement des cordons dunaires de fond de baie, liée à l'absence d'hivers véritablement tempétueux. En outre, la faible pluviométrie des années 2003 et 2005 aurait été favorable à la déflation éolienne sur les estrans et au transport de sédiments vers les hauts de plages et les massifs dunaires. Dans le sud du Finistère, les travaux de B. Hallégouët et A. Hénaff (2006) ont également montré une tendance à la progradation des rivages au cours des dernières années. Pour les auteurs, cette phase de régénération des systèmes littoraux aurait débuté au début des années 1990, succédant à une période d'activité morphogène plus importante entre 1974 et 1990. Les conditions de calme météorologique et océanique des dernières années, marquées par des hivers froids et moins tempétueux, auraient ainsi contribué à diminuer l'importance des processus d'érosion, entraînant la reconstitution naturelle des rivages. Enfin, sur l'île de Banneg en mer d'Iroise, l'analyse des conditions météo-marines menée par S. Suanez et al. (2009) a également montré une diminution de la fréquence des épisodes de hauts niveaux d'eau à partir de 2002, liée à une réduction significative des événements météo-marins de forte intensité. En revanche, la décennie 1980, caractérisée par des hivers plus tempétueux, aurait donné lieu à des épisodes de hauts niveaux d'eau plus fréquents et vraisemblablement plus propices à l'érosion des formes littorales.

Plusieurs éléments peuvent être avancés pour expliquer ces tendances décennales à l'érosion ou à l'engraissement des rivages à l'échelle de la Bretagne. R. Bouligant et P. A. Pirazzoli (1999) ont pu identifier des cycles de 11 à 16 ans dans l'amplitude du phénomène de surcote à Brest qui s'expliquerait par la fréquence plus ou moins forte des vents d'afflux (Pirazzoli, 2000). Selon P.A. Pirazzoli et al. (2004), la périodicité de ces vents pourrait être associée aux variations de l'indice d'Oscillation Nord Atlantique (NAO) qui présente, lui aussi, des cycles d'environ 10 ans (Hurrell, 1995; Hurrell et Deser, 2009). Le passage d'une phase négative à une phase positive de l'indice correspondrait à une augmentation du gradient de pression méridien sur l'Atlantique Nord (Rogers, 1984), induisant un renforcement des vents dominants de direction ouest et sud-ouest en hiver au-delà de la latitude 
$45^{\circ} \mathrm{N}$. Cette configuration suppose donc un déplacement des centres dépressionnaires vers le nord et, de ce fait, une augmentation de l'activité des tempêtes en Bretagne. Sur la figure 10, les principaux épisodes morphogènes recensés sur le Sillon de Talbert au cours des cinquante dernières années par J.-P. Pinot (1994), V. Morel (1997) et B. Latteux (2000) ont été replacés sur la courbe des variations de l'indice NAO hivernal. Il est intéressant de constater que ces événements sont survenus pour l'essentiel lors des phases positives de l'indice NAO. L'épisode de 1987 (fig. 10) est le seul à s'être produit au cours d'une année à NAO négatif. La période de faible activité morphogène entre 2002 et 2006 correspond, par contre, à une phase de faible variation de l'indice, tandis que le renforcement des conditions météo-marines observées à partir de l'hiver 2006-2007 coïncide à nouveau avec une phase positive du NAO. Ainsi, à l'échelle décennale, la variabilité climatique intervenant à l'échelle de l'Atlantique Nord semble constituer un agent de forçage de premier ordre dans l'évolution du Sillon de Talbert, en contrôlant notamment la fréquence et l'intensité des épisodes tempétueux favorables au franchissement du cordon par les vagues lors des pleines mers de vive-eau. Moins probables, des événements extrêmement morphogènes restent néanmoins possibles lors d'une période peu tempétueuse, comme l'illustre l'épisode de 1987.

Il est possible que le renforcement des conditions météo-marines observé sur le Sillon de Talbert durant l'hiver 2006-2007, tout comme l'épisode du 10 mars 2008, marque la fin d'une période de faible activité morphogène en Bretagne et signifie l'amorce d'une nouvelle tendance érosive au cours de la prochaine décennie. Outre les côtes bretonnes, l'ensemble de la façade atlantique française est peut-être affecté par ce changement de cycle morphogénique, comme semble le montrer la tempête Klaus qui a frappé la côte des Landes le 24 janvier 2009, ou encore la tempête Quinten du 10 février 2009 survenue par un coefficient de marée de 105.

Cette étude permet, enfin, d'insister sur le caractère extrêmement spasmodique de la mobilité des cordons de galets, se traduisant par des phases de stabilité plus ou moins longues comme nous l'avons mesuré entre 2002 et 2006 sur le Sillon de Talbert, et de courtes phases de recul par rollover, lors d'événements météo-marins extrêmes tels que l'épisode du 10 mars 2008. Ainsi, les principaux changements morphologiques s'opèrent en quelques heures seulement, dès lors qu'un niveau d'eau élevé à la côte fait porter l'action des vagues au sommet des cordons et permet le déversement de matériel sur le revers. Comme l'a montré S. Costa (2005), le fonctionnement non-linéaire des accumulations de galets s'explique ainsi par des effets de seuil qui caractérisent les processus morphogènes à l'origine du recul des accumulations littorales. Sur les côtes macro-tidales, le dépassement de ces seuils nécessite généralement un contexte météo-marin exceptionnel associant une pleine mer de vive-eau et le passage d'une dépression atmosphérique près des côtes (Costa, 1997; Suanez et Stéphan, 2006; Stéphan, 2008b). Une telle conjonction est relativement rare. L'analyse des niveaux d'eau extrêmes réalisée par P. Stéphan (2008a) sur le Sillon de Talbert permet d'estimer la période de retour de l'épisode du 10 mars 2008 à environ 20 ans. Le long du profil P075, par exemple, les plus hauts jets de rive ont alors dépassé la cote de 14,1 m CM. Un tel niveau d'eau n'avait pas été atteint depuis le mois de janvier 1990 où une violente tempête avait également coïncidé avec une pleine mer de vive-eau.

\section{Conclusion}

Cette étude a montré qu'en favorisant le franchissement d'une large partie du Sillon de Talbert par les vagues de tempête, l'épisode météo-marin du 10 mars 2008 a été responsable d'un transfert massif de sédiments en direction du revers et d'un recul du cordon par rollover d'une dizaine de mètres en moyenne. Ce recul, considérable au regard des volumes sédimentaires mobilisés $\left(120000 \mathrm{~m}^{3}\right)$, contraste avec la relative stabilité du Sillon observée entre 2002 et 2007 . Il permet d'insister sur le caractère très spasmodique de la mobilité des cordons de galets et sur les effets de seuils qui caractérisent les processus morphogènes. Par ailleurs, la tendance post-érosive du Sillon de Talbert à l'exhaussement montre que les cordons de galets sont souvent des systèmes relativement résilients, quand bien même ils connaissent une situation de pénurie sédimentaire. 
Figure 10 : Variations de l'indice NAO hivernal et épisodes les plus morphogènes sur le Sillon de Talbert entre 1950 et 2008

NAO index variations and extrem events counted on Sillon de Talbert between 1950 and 2008

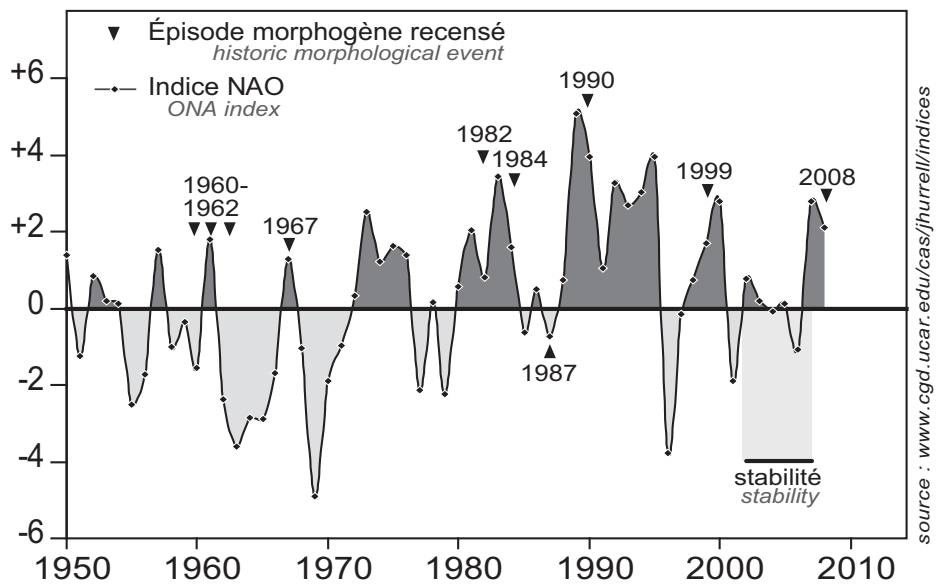

La tempête Johanna est le premier épisode véritablement érosif qu'a connu le Sillon de Talbert depuis la suppression des ouvrages de stabilisation en enrochements, décidée par le Conservatoire du Littoral en 2004. Désormais, la politique de gestion de l'érosion s'appuie sur des interventions ponctuelles visant à accompagner le recul du cordon tout en évitant qu'il se rompe. Après le 10 mars 2008, plusieurs amorces de brèches ont donc été artificiellement colmatées. Toutefois, ces mesures ont été jugées insuffisantes par une partie de la population locale, vraisemblablement traumatisée par les dommages causés par Johanna dans les communes voisines. Précisons, en effet, qu'après la tempête, une quinzaine de demandes de reconnaissance de catastrophe naturelle a été déposée dans le seul département des Côtes-d'Armor. Ainsi, les craintes de voir disparaître le Sillon de Talbert ont resurgi après l'épisode du 10 mars; ces mêmes craintes qui avaient conduit les collectivités locales à mettre en place les enrochements le long du cordon dans les années 1970. Cependant, si les mesures prises par le Conservatoire sont contestées, elles paraissent bien plus adaptées à la dynamique actuelle du Sillon que des ouvrages de stabilisation classiques. Par ailleurs, on peut se réjouir du classement du site en Réserve naturelle régionale en 2006, ce statut imposant désormais que toute intervention anthropique sur le site soit soumise à une réflexion préalable.

\section{Bibliographie}

Battjes J. A., 1974. Surf Similarity, Proceedings of the 14th Coastal Engineering Conference, American Society of Civil Engineers, p. 466-480.

Boersma S. M., Hoenderkamp K., 2003. Tregor, final report IFREMER, Amsterdam, janvier 2003, 25 p.

Bouligand R., Pirazzoli P. A., 1999. Les surcotes et les décotes marines à Brest, étude statistique et évolution, Oceanologica Acta, vol. 22, n 2, p. 153-166.

Buscombe D., Masselink G., 2006. Concepts in gravel beach dynamics, Earth-Science Reviews, vol. 79, p. 33-52.

Butt T., Russell P., 2000. Hydrodynamics and cross-shore sediment transport in the swash-zone of natural beaches: a review, Journal of Coastal Research, vol. 16, n 2, p. 255-268.

Cariolet J.-M., Costa S., Caspar R., Ardhuin F., Magne R., Goasguen G., 2010. Aspects météo-marins de la tempête du 10 mars 2008 en Atlantique et en Manche, Norois. Ce volume.

Carter R. W. G., Johnston T. W., McKenna J., Orford J. D. 1987. Sea-level, sediment supply and coastal changes : examples from the coast of Ireland, Progress in Oceanography, vol. 18, p. 79-101. 
Carter R. W. G., Forbes D. L., Jennings S.C., Orford J. D., Shaw J., Taylor R.B., 1989. Barrier and lagoon coast evolution under differing relative sea-level regimes: examples from Ireland and Nova Scotia, Marine Geology, vol. 88, p. 221-242.

Carter R. W. G., Orford J. D. 1993. The morphodynamics of coarse clastic beaches and barriers: a short- and long-term perspective, Journal of Coastal Research, SI n 15, p. 158-179.

Costa S., 2005. Falaises à recul rapide et plages de galets : de la quantification des dynamiques d'un système complexe à la caractérisation des risques induits, Mémoire d'Habilitation à Diriger des Recherches, Département de Géographie, Université de Bretagne Occidentale, Brest, 310 p.

—, 1997. Dynamique sédimentaire et risque naturel : l’impact des aménagements, des variations du niveau marin et des modifications climatiques entre la baie de Seine et la baie de Somme, Thèse de Doctorat en géographie, Université Paris I, Paris, 376 p.

Forbes D. L., Taylor R. B., Orford J. D., Carter R. W. G., Shaw J., 1991. Gravel-barrier migration and overstepping, Marine Geology, vol. 97, p. 305-313.

Forbes D. L., Orford J. D., Carter R. W. G., Shaw J., Jennings S. C., 1995. Morphodynamic evolution, selforganisation, and instability of coarse-clastic barriers on paraglacial coast, Marine Geology, vol. 126, p. 63-85.

Hallégouët B., HénAfF A. 2006. Évolution récente et gestion des espaces littoraux de l'ouest Cornouaille, Les actes des rencontres de l'ouest Cornouaille "Quelles pistes de développement pour le territoire? ", maijuin 2005, Association Ouest Cornouaille Promotion (AOCP), Pont-L'Abbé, p. 20-34.

Héquette A., Ruz M. H., 1991. Spit and Barrier Island Migration in the Southeastern Canadian Beaufort Sea, Journal of Coastal Research, vol. 7, n³, p. 677-698.

Holland K. T., Raubenheimer B., Guza R. T., Holman R. A., 1995. Runup kinematics on a natural beach, Journal of Geophysical Research, vol. 100, C3, p. 4985-4993.

Holland K. T., Holman R. A., 1993. Statistical distribution of swash maxima on natural beaches, Journal of Geophysical Research, vol. 98, C6, p. 10271-10278.

—, 1996. Field observations of beach cusps and swash motions, Marine Geology, vol. 134, p. 77-93.

Holman R. A., Sallenger A.H., 1985. Setup and swash on a natural beach, Journal of Geophysical Research, vol. 90, C1, p. 945-953.

Holman R. A., 1986. Extreme value statistics for wave run-up on a natural beach, Coastal Engineering, vol. 9, p. $527-544$.

Hurrell J. W., 1995. Decadal trends in the North Atlantic Oscillation: regional temperatures and precipitations, Science, vol. 291, p. 676-679.

Hurrell J. W., Deser C., 2009. North Atlantic climate variability: the role of the North Atlantic Oscillation, Journal of Marine Systems, vol. 78, $\mathrm{n}^{\circ}$ 1, p. 28-41.

Latteux B., 2000. Projet de préservation et de restauration du Sillon de Talbert-Baie de Lanros et ses abords immédiats (commune de Pleubian), $1^{\text {re }}$ partie: Analyse du patrimoine et de ses problèmes, Conservatoire de l'espace littoral et des rivages lacustres, $81 \mathrm{p}$.

Leatherman S. P., 1979. Migration of Assateague Island, Maryland, by inlet and overwash processes, Geology, vol. $7, \mathrm{n}^{\circ} 2$, p. 104-107.

—, 1983. Barrier dynamics and landward migration with Holocene sea-level rise, Nature, vol. 301, p. 415-417.

Masselink G., Li L., 2001. The role of swash infiltration in determining the beachface gradient: a numerical study, Marine Geology, vol. 176, p. 139-156.

Morel V., 1997. De la géomorphologie à la gestion des cordons de galets littoraux de bassin de la Manche et de ses abords : études de cas (Bretagne, Haute-Normandie, sud Angleterre) et réflexions générales, Thèse de doctorat en géographie, Université de Bretagne Occidentale, Brest, 386 p.

Orford J. D., Carter R. W. G., Forbes D. L., 1991. Gravel barrier migration and sea level rise: some observations from Story Head, Nova Scotia, Canada, Journal of Coastal Research, vol. 7, n 2, p. $477-488$.

Orford J. D., Carter R. W. G., McKenna J., Jennings S. C., 1995. The relationship between the rate of mesoscale sea-level rise and the rate of retreat of swash-aligned gravel-dominated barriers, Marine Geology, vol. 124, p. 177-186.

Orford J. D., Carter R. W. G., 1982. Crestal overtop and washover sedimentation on a fringing sandy gravel barrier coast, Carnsore Point, southeast Ireland, Journal of Sedimentary Petrology, vol. 52, n 1, p. $265-278$. 
Pinot J.-P., 1994. Fixer le plan ou gérer le profil : l'exemple du Sillon du Talberv, Cahiers Nantais, n 41-42, p. 307-316.

Pirazzoli P. A., 2000. Surges, atmospheric pressure and wind change and flooding probability on the Atlantic coast of France, Oceanologica Acta, vol. 23, n 6, p. 643-661.

Pirazzoli P. A., Regnauld H., Lemasson L. 2004. Changes in storminess and surges in western France during the last century, Marine Geology, vol. 210, p. 307-323.

Rogers J. C., 1984. The association between the North Atlantic Oscillation and the Southern Oscillation in the Northern Hemisphere, Monthly Weather Review, vol. 112, p. 1999-2015.

Ruessink B. G., Kleinhaus M. G., van den Beukel P. G. L., 1998. Observations of swash under highly dissipative conditions, Journal of Geophysical Research, vol. 103, C2, p. 3111-3118.

Ruggiero P., Komar P. D., McDougal W. G., Marra J. J., Beach R. A., 2001. Wave runup, extreme water levels and the erosion of properties backing beaches, Journal of Coastal Research, vol. 17, n 2, p. 407-419.

StÉPHAN P., 2008a. Les flèches de galets de Bretagne : morphodynamiques passée, présente et prévisible, Thèse de doctorat de Géographie, Université de Bretagne Occidentale, 558 p.

—, 2008b. Mobilité des cordons littoraux et submersions marines. Les flèches de galets de la rade de Brest (Bretagne, France), European Journal of Environmental and Civil Engineering, vol. 12, n 1-2, p. 161-178.

Stockdon H. F., Holman R. A., Howd P. A., Sallenger A. H., 2006. Empirical parameterization of setup, swash and runup, Coastal Engineering, vol. 53, p. 573-588.

Stockdon H. F., Thompson D. M., Fauver A., 2007. Vulnerability of National Park Service Beaches to inundation during a direct hurricane landfall: Cape Lookout National Seashore, US Geological Survey, report 2007-1376, 8 p.

Suanez S., Dehouck A., Stéphan P., 2008. Incertitude de la mesure de terrain en géomorphologie littorale, Approche statistique et quantification des marges d'erreur, dans: Incertitude et environnement, la fin des certitudes scientifiques, P. Allard, D. Fox, B. Picon (dir.), Aix-en-Provence, Edisud, coll. «Écologie Humaine », p. 127-139.

Suanez S., Stéphan P., 2006. Forçages météo-marins et dynamique morphosédimentaire saisonnière des cordons dunaires. Exemple de la baie de Saint-Michel-en-Grève (Côtes-d'Armor, Bretagne), Géomorphologie: Relief. Processus. Environnement, n², p. 91-110.

Suanez S., Fichaut B., Magne R., 2009. Cliff-top storm deposits on Banneg Island, Brittany, France: effects of giant waves in the Eastern Atlantic Ocean, Sedimentary Geology, vol. 220, n 1-2, p. 12-28.

Cet article a été reçu le 30 juin 2009 et définitivement accepté le 3 mars 2010. 
planche V (Pierre STÉPHAN, Serge SUANEZ, Bernard FICHAUT - Franchissement et recul des cordons de galets...)

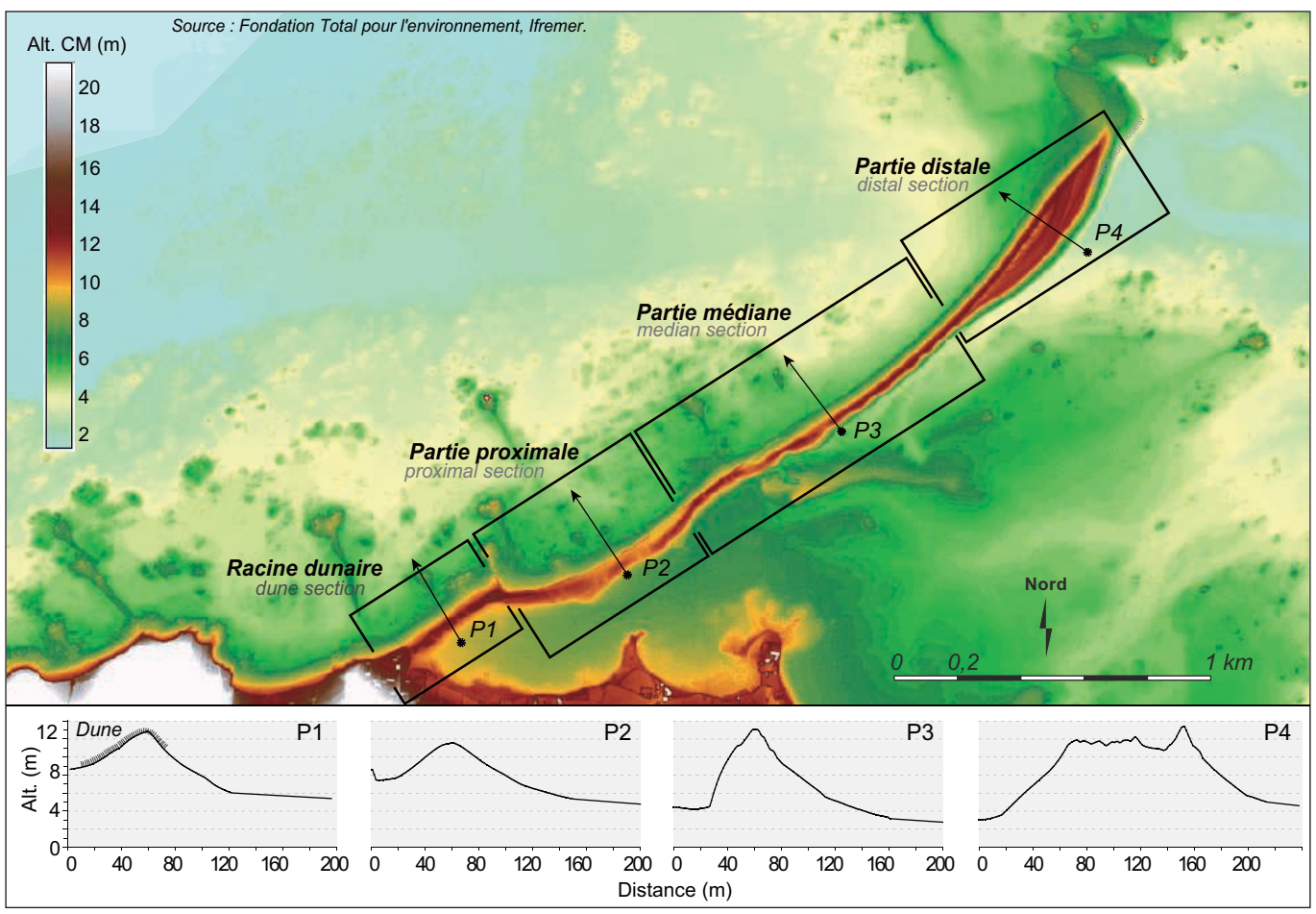

Figure 3 : Levé Lidar d'octobre 2002 et profils topographiques caractéristiques des différentes unités morphosédimentaires du Sillon de Talbert

Lidar topographic survey (October 2002) and transects realised on the different morphosedimentary units of the Sillon de Talbert 